ARTICLE

https://doi.org/10.1038/s41467-019-09522-1

\title{
Network Walking charts transcriptional dynamics of nitrogen signaling by integrating validated and predicted genome-wide interactions
}

Matthew D. Brooks ${ }^{1}$, Jacopo Cirrone ${ }^{2}$, Angelo V. Pasquino (10 ${ }^{1}$, Jose M. Alvarez ${ }^{1}$, Joseph Swift ${ }^{1}$, Shipra Mittal ${ }^{1}$, Che-Lun Juang (1) ', Kranthi Varala ${ }^{3}$, Rodrigo A. Gutiérrez (10 4, Gabriel Krouk (1) ${ }^{5}$, Dennis Shasha ${ }^{2}$ \& Gloria M. Coruzzi (iD ${ }^{1}$

Charting a temporal path in gene networks requires linking early transcription factor (TF)triggered events to downstream effects. We scale-up a cell-based TF-perturbation assay to identify direct regulated targets of 33 nitrogen $(\mathrm{N})$-early response TFs encompassing $88 \%$ of $\mathrm{N}$-responsive Arabidopsis genes. We uncover a duality where each TF is an inducer and repressor, and in vitro cis-motifs are typically specific to regulation directionality. Validated TF-targets $(71,836)$ are used to refine precision of a time-inferred root network, connecting $145 \mathrm{~N}$-responsive TFs and 311 targets. These data are used to chart network paths from direct $\mathrm{TF}_{1}$-regulated targets identified in cells to indirect targets responding only in planta via Network Walking. We uncover network paths from TGA1 and CRF4 to direct $\mathrm{TF}_{2}$ targets, which in turn regulate $76 \%$ and $87 \%$ of $\mathrm{TF}_{1}$ indirect targets in planta, respectively. These results have implications for $\mathrm{N}$-use and the approach can reveal temporal networks for any biological system.

\footnotetext{
${ }^{1}$ Center for Genomics and Systems Biology, Department of Biology, New York University, New York, NY 10003, USA. ${ }^{2}$ Courant Institute for Mathematical Sciences, New York University, New York, NY 10012, USA. ${ }^{3}$ Horticulture and Landscape Architecture/Center for Plant Biology, Purdue University, West Lafayette, IN 47907, USA. ${ }^{4}$ Departamento de Genética Molecular y Microbiología, FONDAP Center for Genome Regulation, Millennium Institute for Integrative Biology, Pontificia Universidad Católica de Chile, Santiago 8331150, Chile. ${ }^{5}$ B\&PMP, CNRS, INRA, Université de Montpellier, Montpellier SupAgro, Montpellier 34060, France. Correspondence and requests for materials should be addressed to G.M.C. (email: gloria.coruzzi@nyu.edu)
} 
$\mathrm{T}$ emporal control of transcriptional networks enables organisms to adapt to a changing environment. Thus, a primary goal of systems biology is to reconstruct the order of transcription factor (TF)-target interactions for the underlying gene regulatory networks (GRNs). To this end, researchers have used de novo network inference to learn GRNs in many organisms spanning microbes, plants, and animals ${ }^{1-3}$. However, a major challenge, especially in higher eukaryotes, is genome-wide validation of the accuracy and predictive power of the resulting GRNs. This is largely due to the lack of methods for rapidly validating the inferred TF-target interactions in vivo ${ }^{1,4}$.

Despite advances in the identification of physical interactions of TFs and targets, there is still relatively little known about which genes are transcriptionally regulated in vivo by a majority of TFs. A recent proliferation of TF-target binding data has emerged from high-throughput in vitro approaches such as DNA affinity purification sequencing (DAP-seq) $)^{5}$, protein binding microarrays $(\mathrm{PBM})^{6}$, and yeast-one-hybrid $(\mathrm{Y} 1 \mathrm{H})^{7}$. However, cis-binding motifs and target genes identified by these methods fail to account for features present in vivo, such as protein-protein interactions, TF combinations, and chromatin structure. Most importantly, TF-target binding data obtained by in vitro methods or by chromatin immunoprecipitation (ChIP) in vivo do not indicate whether the physical TF-target interaction leads to changes in gene expression. Indeed, ChIP is often a poor predictor of TF regulation ${ }^{8-10}$, and is only a snapshot of the most stable TF-binding events under the conditions and time-point assayed ${ }^{11-14}$.

To complement the TF-DNA binding datasets, there is a need for methods to validate TF-target interactions based on a functional assay that takes into account in vivo context. Perturbation of TFs using knockout or overexpressing transgenic lines to identify regulated targets is standard across microbes and higher eukaryotes ${ }^{15-17}$. However, these in vivo approaches are prohibitively time consuming to scale for most eukaryotes. Additionally, it is not possible to distinguish direct from indirect targets in these systems without additional in vivo TF-target binding information, such as ChIP. Moreover, studies across eukaryotes reveal a poor overlap of TF-bound and TF-regulated targets in vivo ${ }^{8-10}$.

To address the need for TF-target assays based on gene expression, we scale-up the throughput of a cell-based temporal $\mathrm{TF}$ perturbation system called TARGET (Transient Assay Reporting Genome-wide Effects of Transcription factors) ${ }^{18}$. This TARGET assay can validate direct TF-target interactions based solely on TF-induced changes in gene expression ${ }^{18-22}$ which overcomes many of the limitations described above. Specifically, the TARGET assay can identify candidate direct TF targets based on gene regulation, is a rapid transient assay, and can be performed on isolated cells from any tissue of interest. Importantly, the TF targets identified by this cell-based assay have also been shown to have in planta relevenace ${ }^{18-21}$. Because cell-based transient expression systems have been developed for many multicellular organisms as a quicker alternative to the creation of stable transgenics and mutants ${ }^{23-25}$, the TARGET approach is broadly applicable. To complement existing genome-wide methods, we apply the TARGET system as a mediumthroughput tool to characterize mechanisms of TF action and improve the available gold standards of functional TF-target interactions for use in network inference.

In this study, we introduce several innovations to scale-up the throughput of the cell-based TARGET system for TF perturbation to $24 \mathrm{TF}$ assays/day (e.g., $8 \mathrm{TFs} \times 3$ replicates). These innovations have allowed us within $\sim 2$ months to identify the candidate direct regulated genome-wide targets of $33 \mathrm{TFs}$ that

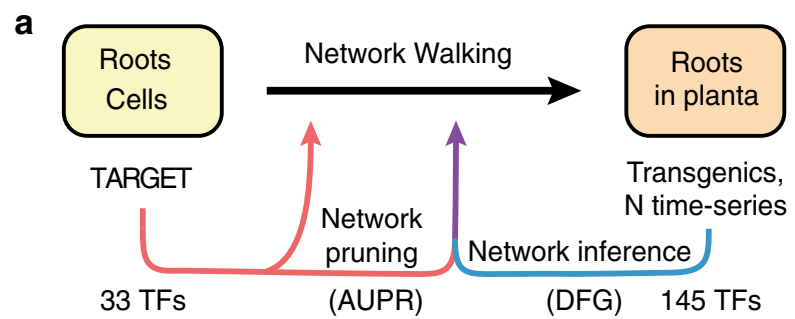

b

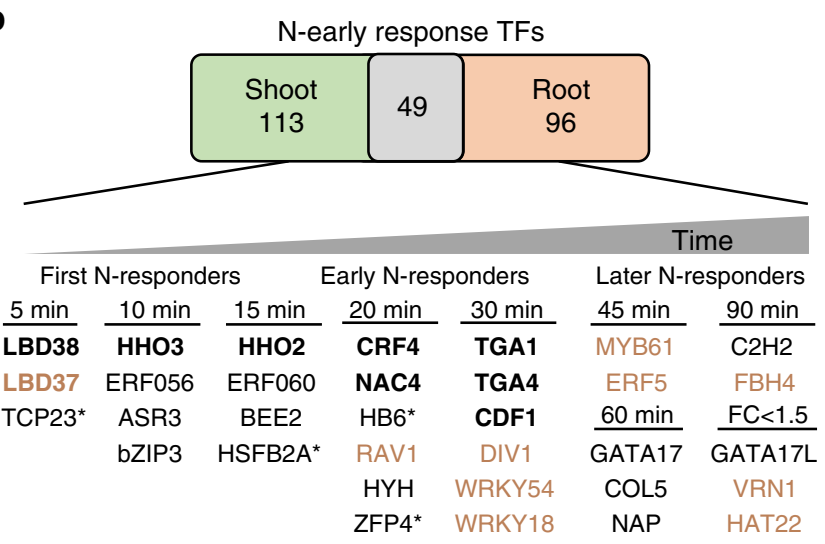

Fig. 1 Network Walking connects validated direct transcription factor (TF) targets to in planta responses. a Schematic overview: the Network Walking approach charts a network path from direct targets of a TF identified in cells to its indirect targets, which only respond in planta. This is achieved using data for 33 TF perturbations in root cells using TARGET (Transient Assay Reporting Genome-wide Effects of Transcription factors $)^{18}$ scaled-up in this study, and a time-series transcriptome of nitrogen $(\mathrm{N})$ response in whole roots $^{29}$. TF-target edges for 145 TFs were inferred using this time-series data in a machine-learning method called dynamic factor graphs (DFG) 48 (blue arrow). The inferred edges were pruned for high-confidence edges (purple arrow) using 71,836 validated edges (red arrow) for 33 TFs in a precision/recall analysis (area under precision recall (AUPR)). The validated edges and high-confidence inferred edges are used to link a TF to its indirect targets in planta via the Network Walk. b The 33 TFs were selected based on their response to $\mathrm{N}$ in shoots and roots (black TFs) or roots only (orange TFs) from the $\mathrm{N}$-treatment time-series data of Varala et al.29. TFs were placed into groups based on their Just-in-Time classification, in which genes were binned based on the first time-point $\mathrm{N}$ treatment caused a fold change of 1.5 relative to the control ${ }^{29}$. TFs in bold have been previously described in the nitrogen response (CRF4 and $\mathrm{CDF}^{29}, \mathrm{NAC4}^{30}, \mathrm{TGA} 1$ and TGA4 ${ }^{26,27}, \mathrm{LBD} 37$ and LBD38 $31, \mathrm{HHO} 2$ and $\mathrm{HHO}^{32}$ ). Asterisk indicates TFs not included in the DFG network as they did not meet the false discovery rate (FDR) threshold

collectively target $\sim 88 \%$ of the genes in the early nitrogen $(\mathrm{N})$ response in Arabidopsis. We use this validated TF-target dataset to define a network path that connects the direct targets of these $\mathrm{N}$-early response TFs in root cells to indirect targets identified only in planta. To do this, we present a Network Walking approach that combines functionally validated (85,144 edges) and time-inferred TF-target edges to connect TF targets validated in root cells, with indirect targets regulated in planta, as shown in Fig. 1a. In our proof-of-concept Network Walking examples, we determine the network path for two known TFs in the $\mathrm{N}$ response, TGA $1^{26-28}$ and $\mathrm{CRF} 4^{29}$. Using this approach, we connect $77 \%$ and $87 \%$ of the indirect targets detected only in planta, back to TGA1 and CRF4, respectively, through intermediate $\mathrm{TF}_{2} \mathrm{~s}$. The Network Walking approach has general application across biological systems. Our proof-of-concept examples have implications for manipulation of networks that 
control plant $\mathrm{N}$-use efficiency, a process that impacts agriculture, the environment, and human health.

\section{Results}

Direct regulated targets of $33 \mathrm{~N}$-early response TFs. We sought to identify which of the 2000 TFs in Arabidopsis facilitate the rapid response to $\mathrm{N}$ signaling in plant roots, as well as the temporal regulatory paths they employ. To do this, we targeted a set of $33 \mathrm{~N}$-early response TFs for functional testing (Fig. 1b), selected based on their rapid transcriptional response to $\mathrm{N}$ treatment in a fine-scale time-course study conducted by Varala et al. ${ }^{29}$. In that study, genes responding to $\mathrm{N}$ treatment as function of time $(\mathrm{N} \times$ Time $)$ were identified by fitting a cubic spline model $^{29}$. These $\mathrm{N} \times$ Time-responsive genes include $145 \mathrm{TFs}$ in roots and 162 TFs in shoots, with an overlap of 49 TFs (Fig. 1b). We selected a subset of 33 TFs that respond across $\mathrm{N} \times$ Time in both shoots and roots, or specific to roots, to validate their $\mathrm{N}$ early response network. This set of 33 TFs includes 9 TFs previously validated in the $\mathrm{N}$ response (e.g., CRF4 and $\mathrm{CDF}{ }^{29}$, NAC4 $4^{30}$, TGA1 and TGA $4^{26-28}$, LBD37 and LBD38 ${ }^{31}, \mathrm{HHO} 2$, and $\mathrm{HHO}^{32}$ ) and $24 \mathrm{TFs}$ with an as yet unknown role in $\mathrm{N}$ signaling. We note that this selection approach misses TFs that only respond post-translationally to the $\mathrm{N}$ signal, such as NLP79,33, a well-known master regulator of the $\mathrm{N}$ response in Arabidopsis.

To determine the genome-wide targets regulated by these 33 $\mathrm{N}$-early response TFs, we used the cell-based TARGET system for inducible TF perturbation in root cells ${ }^{18}$ with our modifications to increase throughput. In the TARGET system, TF nuclear entry is controlled using a subdomain of the glucocorticoid receptor (GR) fused to the TF of interest, an approach that has also been used in planta ${ }^{34,35}$. The GR-TF fusion protein is held in the cytoplasm by HSP90-GR binding, and dexamethasone (DEX) treatment disrupts this interaction, allowing temporal control of TF entry into the nucleus ${ }^{35}$. As has been shown in planta ${ }^{35}$ and in isolated roots cells ${ }^{18}$, pre-treatment with cycloheximide (CHX) blocks downstream regulation of secondary TF targets. Thus, candidate direct TF targets can be identified as those that respond transcriptionally to DEX-induced TF nuclear import in the presence of $\mathrm{CHX}^{18,35}$.

In our study, we made two innovations that increased the throughput of the TARGET assay for TF perturbation: (i) the use of an empty vector (EV) control, and (ii) pooling of cells separately transfected with vectors containing either red fluorescent protein (RFP) or green fluorescent protein (GFP), prior to fluorescence-activated cell sorting (FACS) selection of positively transfected cells (see Methods and Supplementary Fig. 1a). Both changes enabled us to increase the throughput of TF perturbations screened via TARGET up to $24 \mathrm{TF}$ assays/day (e.g. $8 \mathrm{TFs} \times 3$ replicates). Additionally, in this design, because all samples are treated with CHX (e.g., TFs and EV), it circumvents the need to compare $\pm \mathrm{CHX}$ samples, which may impact gene expression and the ability to identify TF-regulated genes (also see Supplementary Fig. 2, Supplementary Data 1, and Supplementary Methods). Using this enhanced medium-throughput TARGET approach, we could identify direct regulated targets of 33 TFs within $\sim 2$ months (Fig. 2). To obtain a list of genes differentially expressed (DE) in response to TF perturbation, we performed RNA-seq on root cells collected $3 \mathrm{~h}$ after DEX-induced nuclear entry of the GR-TF fusion. We then compared the transcriptome for each of the 33 TF samples (performed in triplicate) to the EV-negative control using the bioinformatics analysis pipeline shown in Supplementary Fig. 1b. The TF targets identified as DE between each of the $33 \mathrm{TFs}$ and EV control (false discovery rate $(\mathrm{FDR})<0.05$ ) are reported in Supplementary Data 2 and represent 85,144 TF-target interactions. Further details on the treatments can be found in Supplementary Figs. 3 and 4.

The number of candidate direct regulated targets for each of the $33 \mathrm{~N}$-early response TFs identified in our enhanced TARGET approach ranged between a low of $417 \mathrm{DE}$ genes (VRN1) to a high of $6028 \mathrm{DE}$ genes (HSFB2A) (Fig. 2). This range in the number of TF targets was not related to differences in TF overexpression level, as there was no correlation between the level of TF expression (compared to EV baseline) and the number of TF-regulated targets (Supplementary Fig. 5). Additionally, we found that there are typically fewer direct regulated targets for a TF detected using TARGET, an in vivo assay, compared to the number of TF-bound targets identified in vitro by DAP-seq ${ }^{5}$ (Supplementary Fig. 6). Indeed, we show that our TARGET data can be used to refine in vitro TF-DNA binding edges to identify which TF-target interactions are likely to result in gene regulation within a plant cell. We found that only a fraction of TF-bound targets from DAP-seq ${ }^{5}$ are regulated by the $\mathrm{TF}$ in our root protoplast TARGET assay (Supplementary Fig. 6). However, we did uncover a significant enrichment of direct TF-regulated targets that are also TF-bound in vitro for 13/17 TFs with DAPseq data ${ }^{5}$ (Supplementary Data 3 ).

To assess how direct regulated TF targets identified compare with TF targets identified in planta, we examined available in planta ChIP binding data for three TFs, WRKY18 ${ }^{36}$, HB6, and HAT22 ${ }^{37}$, and found large and significant overlap in each case, despite different experimental growth and treatment conditions (Table 1). Lastly, for TGA1, a well-studied TF in N signaling ${ }^{26-28}$, we observed a large and highly significant overlap (600 genes, $p$ value $=1.78 \mathrm{E}-19$, Fisher's exact test) of direct regulated targets identified in root cells using the TARGET system, compared to DE genes resulting from TGA1 overexpression in roots of whole plants (Supplementary Data 4 and Supplementary Fig. 7) (see Methods). These results collectively support that the candidate direct TF targets identified using the TARGET assay in root cells are enriched in bona fide targets with in planta relevance.

$\mathrm{N} \times$ Time genes enriched in direct regulated targets of 33 TFs. The $33 \mathrm{~N}$-early response TFs were selected based on their $\mathrm{N} \times$ Time response in shoots and roots, or roots only, from the study by Varala et al. ${ }^{29}$ (Fig. 1b). We therefore examined whether the direct regulated targets of each TF identified in TARGET overlapped with $\mathrm{N} \times$ Time-responsive genes in shoots or roots of whole plants. To do this, we calculated the N-response specificity for each TF by determining the percent of the target genes for a TF that are also $\mathrm{N} \times$ Time-responsive genes in roots or shoots of whole plants (Fig. 2, see Methods). We also determined the influence of each $\mathrm{TF}$ on the $\mathrm{N} \times$ Time genes (i.e., the percent of $\mathrm{N} \times$ Time genes regulated by a particular $\mathrm{TF}$ ) for each organ (Fig. 2, see Methods). The 33 TFs in Fig. 2 were ranked based on the $\mathrm{N}$ specificity Index ${ }^{29}$ of their validated targets (shown by color shading), a measure of the significance of the influence a TF has on the $\mathrm{N} \times$ Time genes in each organ (see Methods). Overall, the targets of each of the 33 TFs significantly overlapped with the $\mathrm{N} \times$ Time genes in shoots and/or roots of whole plants (Fig. 2). However, the ranking of TFs was organ specific. For instance, CRF4, a known TF in the $\mathrm{N}$ response in shoots ${ }^{29}$, and ERF5 (Fig. 2, green arrows) are examples of TFs whose direct regulated targets showed organ specificity for the shoot $\mathrm{N} \times$ Time genes. Conversely, the targets of NAP and the known N-response regulator LBD37 $7^{31}$ (Fig. 2, orange arrows) showed specificity for root $\mathrm{N} \times$ Time genes (Fig. 2, brown arrows). Finally, TFs that our study now implicates in the $\mathrm{N}$ response, e.g., bZIP3 and RAV1, controlled a significant number of genes that respond to $\mathrm{N}$ treatment in both shoots and roots (Fig. 2, black arrows). 


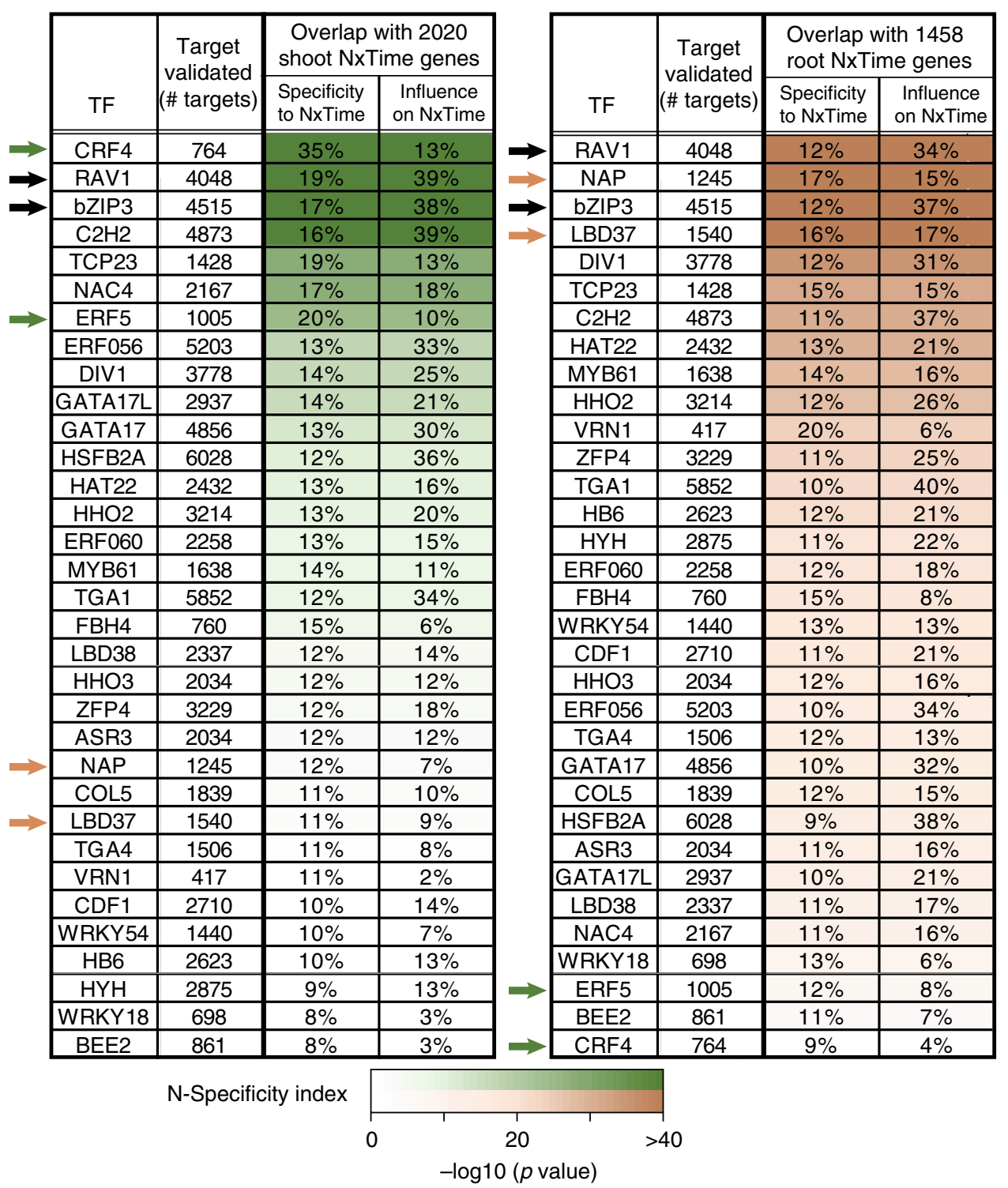

Fig. 2 Validated direct targets of the 33 nitrogen $(N)$-early response transcription factors (TFs) are enriched in $\mathrm{N} \times$ Time genes. The intersection of direct regulated targets for the $33 \mathrm{~N}$-early response TFs identified in root cells using the TARGET (Transient Assay Reporting Genome-wide Effects of Transcription factors) system with $\mathrm{N} \times$ Time genes from $V$ arala et al. ${ }^{29}$. This allowed identification of TFs regulating a significant portion of the $\mathrm{N}$ response in both roots and shoots (e.g., bZIP3/RAV1, black arrows). The direct regulated targets of other TFs are enriched in organ-specific N $\times$ Time response genes. These include CRF4/ERF5, which are specifically enriched for the shoot $\mathrm{N} \times$ Time response genes (green arrows), and NAP/LBD37, which are specifically enriched for the root $\mathrm{N} \times$ Time response genes (orange arrows). Green and orange shading represents the $\mathrm{N}$ specificity Index ${ }^{29}$, the $p$ value calculated using the one proportion z-test (see Methods)

Table 1 Direct regulated TF targets from cells significantly overlap with in planta TF binding

\begin{tabular}{llllll} 
TF & $\begin{array}{l}\text { Direct regulated targets in root } \\
\text { cells (TARGET, this study) }\end{array}$ & $\begin{array}{l}\text { In planta-bound targets } \\
\text { (ChIP36,37) }\end{array}$ & $\begin{array}{l}\text { No. of genes } \\
\text { in overlap }\end{array}$ & $\begin{array}{l}\text { \% of genes } \\
\text { in overlap }\end{array}$ & $\begin{array}{l}\boldsymbol{p} \text { value (Fisher's } \\
\text { exact test) }\end{array}$ \\
\hline HAT22 & 2432 & 5902 & $1035 / 2432$ & $43 \%$ & $5.6 \mathrm{e}-56$ \\
HB6 & 2623 & 7503 & $1270 / 2623$ & $48 \%$ & $3.1 \mathrm{e}-42$ \\
WRKY18 & 698 & 805 & $159 / 698$ & $23 \%$ & $3.8 \mathrm{e}-78$ \\
\hline TF transcription factor, TARGET Transient Assay Reporting Genome-wide Effects of Transcription factors, ChIP chromatin immunoprecipitation & \\
\hline
\end{tabular}

TF-target edges validate a network regulating $\mathrm{N}$ processes. The above TARGET results ranked the $33 \mathrm{~N}$-early response TFs according to their individual roles in regulating genes in the $\mathrm{N}$ responses in planta (Fig. 2). We next asked how this set of TFs work together in mediating the $\mathrm{N}$ response. Collectively, these 33 TFs regulate $88 \%$ of the $\mathrm{N} \times$ Time geneset in roots $(1288 /$ 1458 genes; $p$ value $=1.55 \mathrm{E}-67$, Fisher's exact test), and $88 \%$ of the $\mathrm{N} \times$ Time geneset in shoots $(1785 / 2020 ; p$ value $=5.65 \mathrm{E}-45$ 


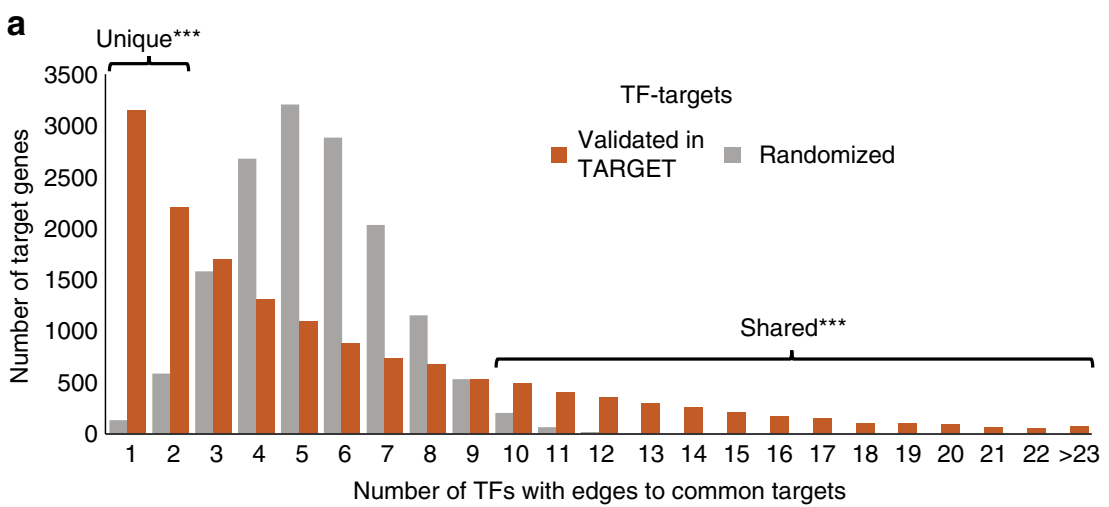

b

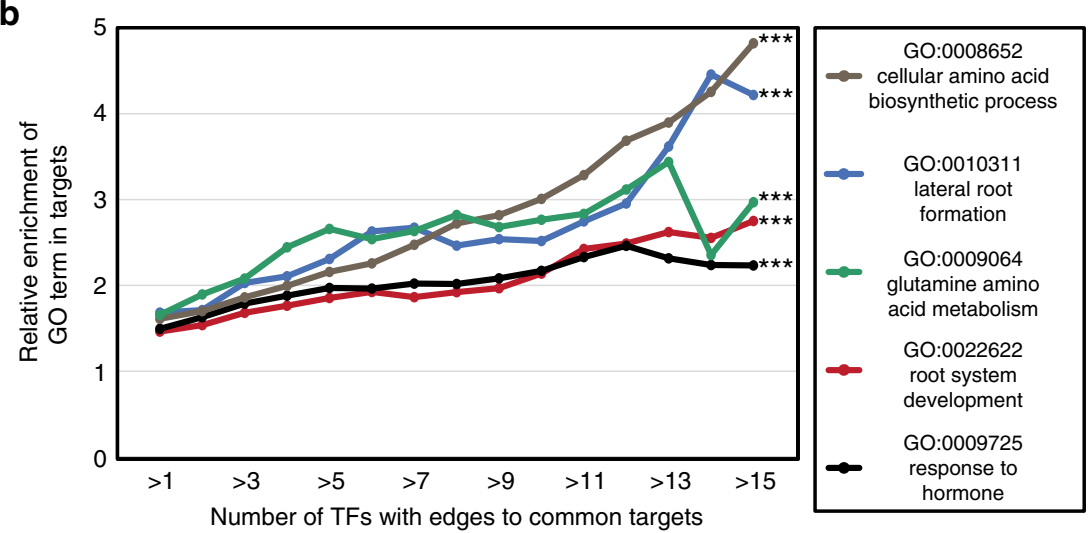

Fig. 3 Nitrogen (N)-early response transcription factors (TFs) coordinate a connected network of N-related genes. a TF-target edges identified by TARGET (Transient Assay Reporting Genome-wide Effects of Transcription factors) in root cells for the $33 \mathrm{~N}$-early response TFs were used to construct a validated network (orange bars). This validated TF-target network resembles a scale-free distribution, with significantly more unique targets (edges to 1 or 2 TFs) or shared targets (edges to 10 or more TFs), compared to a network which contains the same TFs and targets but with randomized edges (gray bars) ( $n=$ 1000). b Enrichment of gene ontology (GO) terms for N-related processes increases as the number of TFs regulating the set of target genes increases. Significance was tested by calculating a figure of merit, called Focus, for the validated TF-target network and comparing it to the Focus values of randomized networks $(n=1000)$ in which the edges within the validated network were shuffled. ( ${ }^{\star \star \star} p$ value $<0.001$, permutation test) (see Methods). Source data of Figs. $3 a$ and $3 b$ are provided as a Source Data file

Fisher's exact test) (Supplementary Table 1). To gain further insight into their collective influence, we explored the network topology for the $33 \mathrm{TFs}$ and their genome-wide targets. To do this, we compared the distribution of TF-target edges within the validated TARGET network for the 33 TFs (Fig. 3a, orange bars), to a network that contains the same TFs and targets but with randomized edges (Fig. 3a, gray bars). The distribution of edges in the validated TARGET network differs significantly from the randomized network. Specifically, compared to the random network, the validated TF-target network contains significantly ( $p$ value $<0.001$, permutation test) more targets that were unique (targeted by only one or two of the TFs) as well as shared (targeted by $\geq 10 \mathrm{TFs}$ ) (Fig. 3a).

We next asked whether the shared targets of the $33 \mathrm{~N}$-early response TFs are enriched for $\mathrm{N}$-related processes. To test this, we calculated the enrichment for Gene Ontology (GO) terms (see Methods). This analysis showed that the collective targets of the 33 TFs were enriched in GO terms such as: $\mathrm{N}$ compound metabolic process, cellular amino acid biosynthesis, lateral root formation, and response to hormone (Supplementary Data 5). Additionally, enrichment of N-related GO terms increased as the number of TFs with edges to a set of common targets increased (Fig. 3b). To test whether this increased enrichment is significant, we devised a figure of merit, which we call Focus (see Methods). The Focus for a TF-target network is greater with respect to set of genes (e.g., GO term) when TFs have more edges to that set of genes. This test determines the probability that the Focus calculated from the edges in the TARGET validated network is higher than we would expect to see by chance. For the network of all validated targets of the $\mathrm{N}$-early response TFs, the Focus for each of the GO terms was significantly greater than for the randomized networks ( $p$ value $<0.001$, permutation test) (Fig. 3b).

We also examined how each of these 33 TFs influence the expression of genes involved in $\mathrm{N}$ use, including $\mathrm{N}$ uptake and assimilation, by plotting a heatmap of the effect each TF had on the expression of these genes (Supplementary Fig. 8). Overall, this set of 33 TFs regulated genes involved in $\mathrm{N}$ use more at the level of $\mathrm{N}$ reduction and assimilation, compared to $\mathrm{N}$ uptake/ transport (Supplementary Fig. 8). This finding is consistent with the high enrichment of the cellular amino acid biosynthesis GO term in the shared targets of the 33 TFs (Fig. 3b). These TARGET results can also help define which edges detected from in vitro TF-DNA binding experiments may lead to functional gene regulation. Specifically, our results suggest that TF target binding studies may underestimate $\left(\mathrm{Y}_{1} \mathrm{H}^{28}\right)$, or overestimate $(\text { DAP-Seq })^{5}, \mathrm{TF}-$ target interactions within the $\mathrm{N}$-metabolism network, in comparison to the functionally regulated TF targets we identified in root cells using the TARGET assay (Supplementary Fig. 9). 


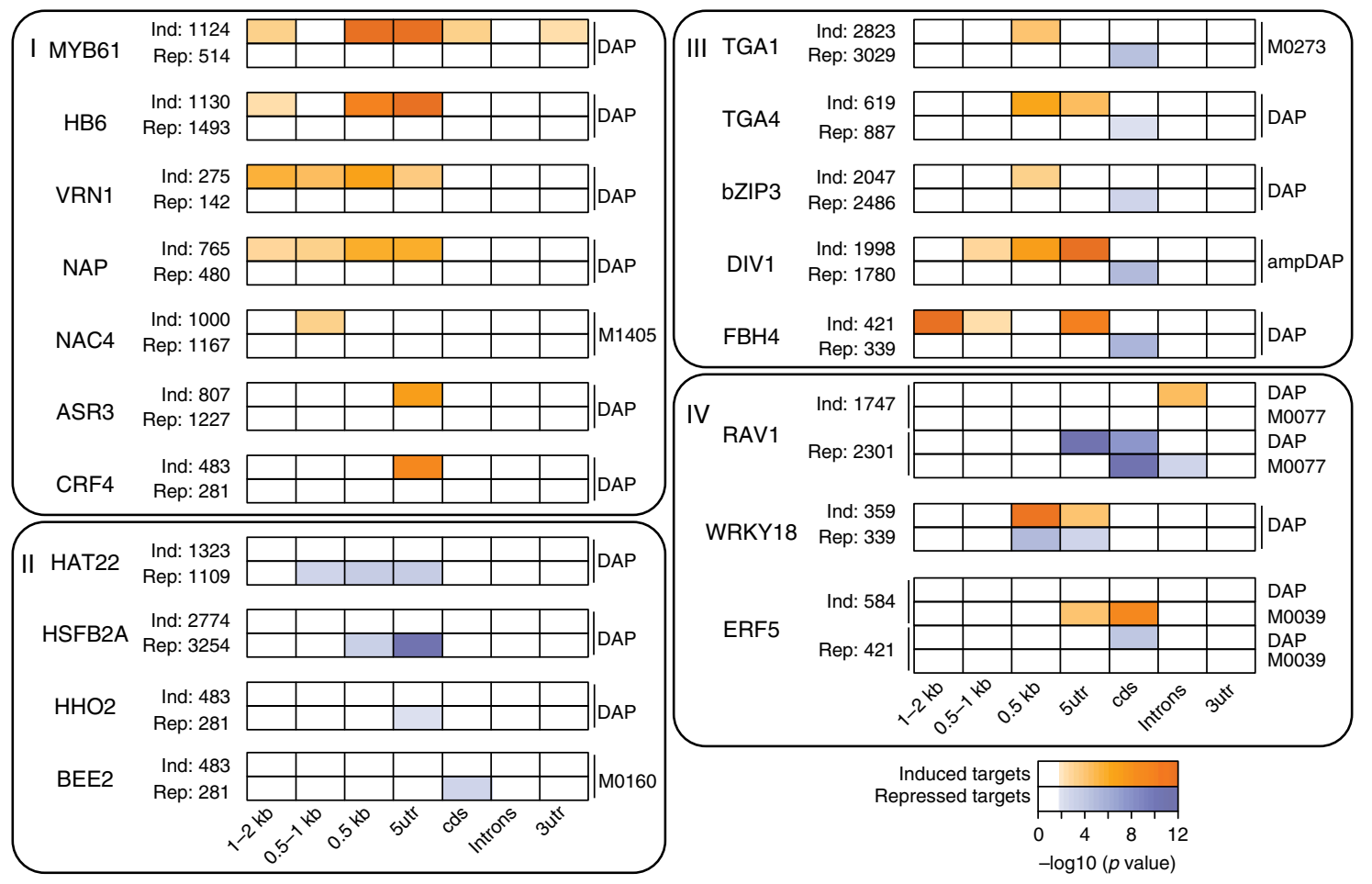

Fig. 4 Cis-motif enrichment in distinct genic regions is associated with transcription factor (TF) induction or TF repression. TF perturbation assays of the 33 nitrogen (N)-early response TFs in root cells reveals that each TF can act both as an inducer and a repressor of direct target genes (number of targets indicated to the right of TF name). Enrichment analysis revealed that TF cis-binding motifs are often specific to a particular type of regulation (e.g., induction vs. repression). Cis-motif data available for 21/33 TFs enabled us to classify these TFs into four groups. Targets identified by the cell-based TARGET (Transient Assay Reporting Genome-wide Effects of Transcription factors) assay uncovered TFs whose known cis-motifs are associated with induction of targets (group I) and TFs with cis-motifs associated with repression of targets (group II). Another group of TFs (group III), including all the bZIP family members in this study, showed cis-motif enrichment in the promoter or 5' untranslated region (UTR) of induced targets, while repressed targets only were enriched for the cis-motif in the coding sequence (CDS). The final set of TFs (group IV) showed enrichment of their known cis-motifs in the overlapping regions of both induced and repressed targets. $\mathrm{HHO} 3$ and TCP23 showed no enrichment of their cis-motifs in any region and are not shown. One or two representative motifs for each TF from the following sources are shown: DNA affinity purification sequencing (DAP-seq) ${ }^{5}$ with genomic DNA (DAP), DAP-seq with PCR-amplified gDNA (ampDAP), and Cis-BP38 (labeled with motif ID). Orange shading (induced targets) and blue shading (repressed targets) represents the enrichment of motifs as calculated using the AME tool within the MEME suite ${ }^{69}$, with $p$ values determined using Fisher's exact test. Results for all cis-motifs can be found in Supplementary Data 6

Cis-motifs for a TF are linked to induction or repression. We next used our TARGET data on the direct regulated targets of the $33 \mathrm{~N}$-early response TFs to filter the in vitro TF-DNA binding data for functional regulation in vivo. Notably, each of the 33 TFs acted as both an inducer or as a repressor of distinct sets of target genes (Fig. 4 and Supplementary Data 2). Because of this duality of TF function, we were able to classify known cis-binding motifs based on their association with the direction of gene regulation, e.g., induction or repression. We performed this analysis for the $21 / 33$ TFs that have cis-binding motif data from DAP-seq ${ }^{5}$ (34 cis-motifs), Cis-BP ${ }^{38}$ (16 cis-motifs), $\mathrm{PBM}^{39}$ (3 cis-motifs), or in vivo $\mathrm{ChIP}^{37}$ (4 cis-motifs). We searched for enrichment of these known cis-motifs for each TF in distinct gene regions of induced or repressed direct regulated targets for each TF (Fig. 4, see Methods). For 19/21 TFs and 50/57 cis-motifs, we found a significant enrichment (FDR $<0.05$, Fisher's exact test) of at least one cis-motif in at least one gene region in the direct TF-regulated targets. When we used all regulated TF targets combined (e.g., induced and repressed), only 15/21 TFs in our study and 40/57 unique cis-motifs showed cis-motif enrichment (Supplementary Data 6).

Typically, we detected enrichment of known cis-motifs for each TF in the proximal promoter or 5' untranslated region (UTR) (Fig. 4). However, for some cis-motifs, we also found enrichment in other gene regions such as the coding sequence (CDS), introns, or 3'UTR (Fig. 4), which has been previously reported ${ }^{5,40}$. Unexpectedly, for $11 / 21 \mathrm{TFs}$, their known cis-motif was enriched exclusively in either induced TF targets (group I) or repressed TF targets (group II) (Fig. 4). Another unexpected finding was that a set of 5 TFs (group III) showed enrichment of their cis-binding motif in the promoter and 5'UTR for induced targets; however, for repressed targets, the cis-motif was enriched only in the CDS (Fig. 4). Lastly, three transcription factors, RAV1, WRKY18, and ERF5 (Group IV), displayed enrichment of their cis-binding motifs in the same overlapping region for both induced and repressed targets (Fig. 4). We note that the results of our cisbinding motif enrichment analysis were similar when we first filtered for accessible chromatin in the $500 \mathrm{bp}$ promoter, as determined in Arabidopsis roots by DNase I hypersensitivity 40 (Supplementary Data 7). Additionally, the above cis-motif enrichment results were also supported when in vitro TF binding $\left(\mathrm{DAP}-\mathrm{seq}^{5}\right)$ are intersected with the induced and repressed direct regulated TF targets (Supplementary Data 3).

We next addressed whether the direct regulated TF targets that are not enriched in a known cis-binding motif for that TF physically associate with the TF in vivo. To do this, we intersected the induced and repressed regulated targets with in planta-bound targets identified using available ChIP-seq data for HB6 and HAT $22^{37}$ (Supplementary Table 2). For both of these TFs, the induced and repressed direct regulated TF targets overlapped 
significantly with the in planta TF-bound targets (Supplementary Table 2). This result suggests that either there is an as yet unidentified secondary cis-motif or that the TF binds to the target via TF-TF interactions. The latter interpretation is supported by the identification of TF partner elements described below.

Partner TF cis-motif clusters are enriched in TF targets. We next sought to identify putative TFs partners that may work together with the $33 \mathrm{~N}$-early response TFs to coordinate gene regulation in the dynamic $\mathrm{N} \times$ Time response network. To do this, we looked for enrichment of cis-motifs for any TF in direct regulated targets of the $33 \mathrm{TFs}$. Given the large number of plant TF-binding cis-motifs from high-throughput methods such as DAP-seq ${ }^{5}$ and $\mathrm{PBM}^{38,39}$, and the fact that TFs from the same family often have similar cis-motifs, searching for each of the 1282 available cis-motifs is impractical. Therefore, we used the RSAT matrix-clustering tool ${ }^{41}$ on all of these known cis-motifs and identified 80 cis-motifs clusters (Supplementary Fig. 10). Cismotifs from TFs belonging to the same family generally fell into the same cis-motif cluster, as seen previously for smaller sets of motifs ${ }^{5,42}$. For each cis-motif cluster, we obtained a consensus cismotif (CCM) and corresponding position weight matrix (PWM $)^{41}$ (Supplementary Data 8 and 9).

Using the PWM for each of the 80 cis-motif clusters, we looked for enrichment of each CCM in the 500 bp promoter (Fig. 5) and gene body (Supplementary Fig. 11) of the induced vs. repressed direct regulated targets of the $33 \mathrm{TFs}$. This analysis uncovered cis-motif enrichment in at least one of these regions for $30 / 33$
TFs. Often, an enriched CCM represented a cluster for a TF family different from the TF tested in TARGET itself, pointing to the involvement of putative TF-TF interactions in gene regulation. This hypothesis is supported by validated protein-protein TF interactions ${ }^{43-47}$ between several of the 33 TFs assayed in TARGET and TF family members from the other cis-motif groups revealed by CCM enrichment analysis (Fig. 5 and Supplementary Fig. 11, black circles).

Functional validation of a time-inferred $\mathrm{N}$-response network. We next sought to expand our GRN of the $\mathrm{N} \times$ Time response in roots beyond the direct regulated targets of the $33 \mathrm{~N}$-early response TFs validated using TARGET (Fig. 2). To do this, we used our validated TF-target data to refine the precision of a GRN predicted from fine-scale time-series transcriptome data of $\mathrm{N}$ treatment in roots ${ }^{29}$ using dynamic factor graphs $(\mathrm{DFG})^{48}$. DFG is a machine-learning method that can use time-series data to estimate the quantitative influence of TFs at time $t$ on target genes at time $t+1^{48}$. This DFG approach has been used to learn network models that can predict gene expression states at future time points, even when few time points are tested ${ }^{29,49}$. In our application, the resulting DFG predictions provided an edge score, or measure of influence, for 145 TFs on every target gene in the root $\mathrm{N} \times$ Time network, totaling 211,410 TF-target edges.

To refine these time-based TF-target predictions, we used 71,836 validated TF-target edges for $29 / 33$ TFs to calculate a precision threshold for the DFG predicted edges. This enabled us to set an edge score to prune the DFG network ${ }^{1}$ and retain only a

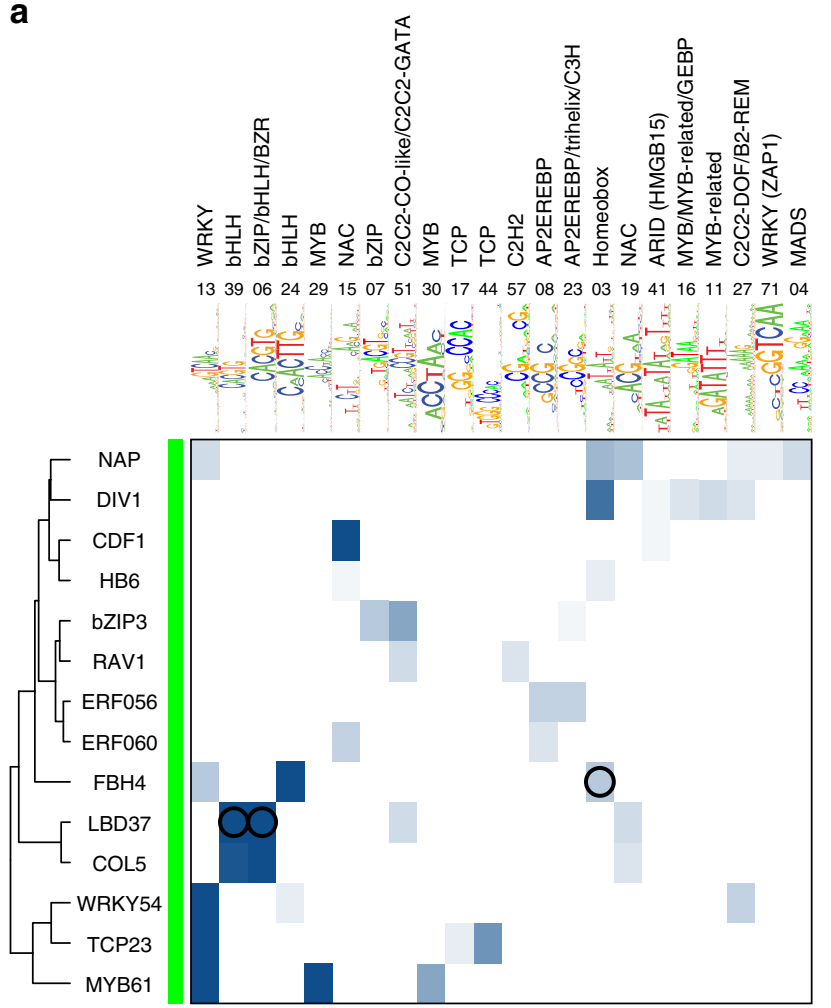

b

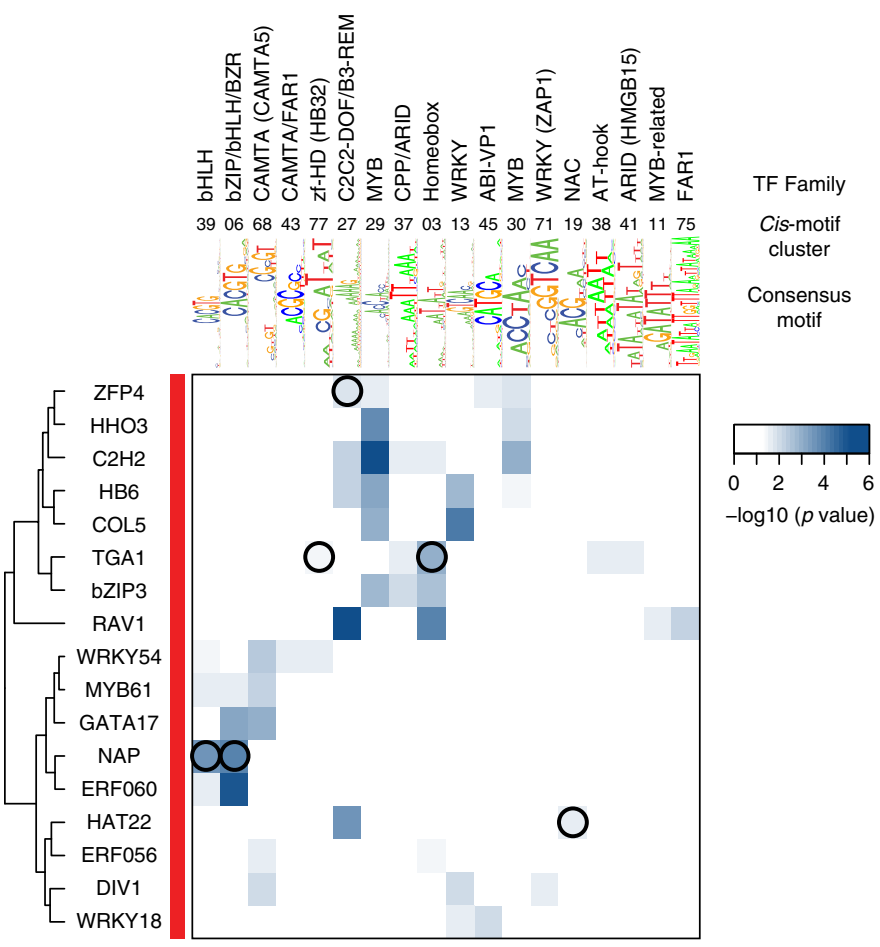

Fig. 5 Direct regulated transcription factor (TF) targets are enriched for cis-motif clusters of partner TFs. Heatmap of enriched consensus cis-motifs for the 80 cis-motif clusters (columns) in the 500 bp promoter of the $\mathbf{a}$ induced and $\mathbf{b}$ repressed direct regulated targets of the 33 nitrogen ( $\mathrm{N}$ )-early response TFs. The consensus cis-motif (CCM) logo, cluster number, and family representation for each CCM is shown above. Instances where there is a validated interaction between the TF under study and another TF within a family represented by the enriched CCM cluster are marked by a black circle. Of the 33 TFs validated in TARGET, only TFs with enrichment of any of the 80 CCMs in the 500 bp promoter are shown. Cis-binding motif clusters were determined using cis-motifs for Arabidopsis transcription factors collected from DNA affinity purification sequencing (DAP-seq) ${ }^{5}$, Cis-BP38, and protein binding microarrays $(\mathrm{PBM})^{39}$. Blue shading represents $p$ values calculated using Fisher's exact test and false discovery rate (FDR) corrected. Source data of Fig. $5 a, b$ are provided as a Source Data file 
high-confidence $\mathrm{TF}$-target edges involved in the root $\mathrm{N}$ response. Four of our 33 TFs-ZFP4, HSFB2A, TCP23, and HB6-were excluded from this analysis, as they did not meet the stringent threshold $(F D R<0.01)$ used to select the $\mathrm{N} \times$ Time genes for DFG predictions ${ }^{29}$. The results of this precision/recall (PR) analysis showed that the area under precision recall (AUPR) for the TF-target predictions in the DFG predicted GRN (0.2372) was significantly greater than for 1000 random PR curves $($ mean $=$ 0.1948 ) (Fig. 6 and Table 2). From the PR curve generated using the validated edges of 29 TFs, we chose a precision threshold cutoff of 0.32 - the point at which the curve begins to flatten out-to filter the GRN for high-confidence TF-target edges. Our precision cut-off score of 0.32 (i.e., $\sim 1 / 3$ rd of predicted edges are validated) is of comparable scale to the 0.50 precision achieved using an ensemble approach of multiple network inference methods in simpler microbial systems ${ }^{1}$. At this precision cut-off, the resulting pruned GRN was comprised of 6863 total high-confidence edges between 145 TFs and 311 targets in the root $\mathrm{N} \times$ Time response network (Table 2, Supplementary Fig. 12 and Supplementary Data 10).

To evaluate the individual contribution of each TF to the edge pruning in the GRN, we calculated precision, recall, and F-score (harmonic mean of precision and recall ${ }^{50}$ ) for each of the 29 TFs within the pruned DFG network individually (Supplementary Table 3). While there was variation in all three metrics for each of the $29 \mathrm{TFs}$, the mean precision, recall, and F-score among all 29 TFs was $0.393,0.149$, and 0.17 , respectively (Supplementary Table 3). These values are close to weighted values for the precision, recall, and $F$-score calculated from the TARGET edges for all 29 TFs combined (Supplementary Table 3). This result indicates that the collective PR measures are not biased towards a few TFs with many edges. Importantly, the $95 \%$ confidence intervals for precision $(0.320-0.465)$, recall $(0.064-0.204)$, and $F$-score $(0.14-0.20)$ indicate that the TF-target edges predicted by DFG for the remaining $116 \mathrm{TFs}$ in the $\mathrm{N} \times$ Time network and their 311 targets are also likely to be true for $\sim 1 / 3$ rd of the highconfidence TF-target edge predictions.

Network Walking charts paths from direct to indirect targets. Finally, we performed an analysis that integrates the validated TF-targets edges for $33 \mathrm{~N}$-early response TFs from TARGET with the high-confidence edges for the 116 untested TFs in the pruned GRN and in planta TF perturbation data. In an approach called Network Walking (Fig. 7a), we used these combined datasets to chart a path for a $\mathrm{TF}_{1}$ from its direct regulated targets in root cells, to its indirect regulated targets in planta via intermediate $\mathrm{TF}_{2} \mathrm{~s}$. As proof of concept, we demonstrated how Network Walking revealed the network paths and mode of action for two important regulators of the $\mathrm{N}$ response in planta-TGA1 $1^{26-28}$ and CRF4 ${ }^{29}$ (Fig. 7b, c). The TF perturbation data used in this Network Walking analysis included direct targets that respond to the TF in cells (e.g., in TARGET) (Supplementary Data 2), and those that respond to TGA1 (this study, Supplementary Data 4) or CRF4 overexpression in planta (Varala et al. ${ }^{29}$, Supplementary Data 11).

In Network Walking, the first step is to use the direct regulated targets of $\mathrm{TF}_{1}$ (e.g., TGA1 or CRF4) identified using the TARGET assay to identify which $\mathrm{DE}$ genes from in planta perturbation are direct vs. indirect $\mathrm{TF}_{1}$ targets. In the TGA1 example, the TARGET assay showed that TGA1 directly regulated 580 root $\mathrm{N} \times$ Time genes (Fig. 7b, yellow box), including 104 direct target genes that also respond to TGA1 overexpression in planta. The second step is to connect a path from $\mathrm{TF}_{1}$-via a $\mathrm{TF}_{2}$ - to the $\mathrm{TF}_{1}$ indirect targets which only respond in planta. To connect TGA1 to its indirect targets, we used validated $\mathrm{TF}_{2}$ direct target edges from TARGET assays (Fig. 2), as well as the high-confidence

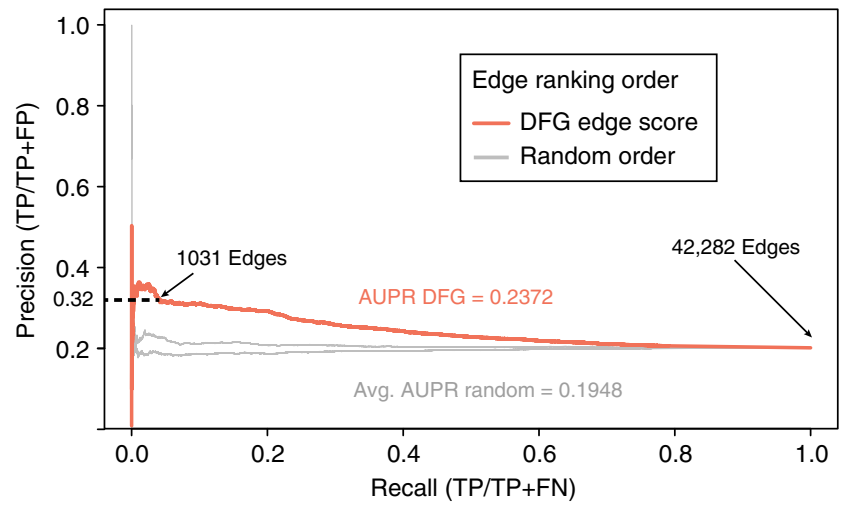

Fig. 6 Validation of a time-inferred network using area under precision recall analysis. Genome-wide regulated targets of 29 transcription factors (TFs) captured in root cells using the TARGET (Transient Assay Reporting Genome-wide Effects of Transcription factors) system (Fig. 2, Supplementary Data 2) were used to calculate the precision and recall of the dynamic factor graph (DFG) inferred gene regulatory network (GRN) based on $\mathrm{N} \times$ Time response genes in roots. Area under precision recall (AUPR) analysis demonstrates that the ranking of edges in the DFG time-inferred network (orange line) is significantly better ( $p$ value $<0.001$, permutation test) than randomizing the order of edge rankings $(n=1000)$ (gray lines represent random networks with highest and lowest AUPR). From this plot a 0.32 precision cut-off was chosen as the highest value before the curve flattens. TP true positives, FP false positives, FN false negatives. Source data are provided as a Source Data file

Table 2 Precision and recall pruning of a time-inferred network using TF-target validation

\begin{tabular}{|ll|} 
Validated network measures & Value \\
\hline Area under precision recall (validated network) & 0.2372 \\
Area under precision recall (random network average) & 0.1948 \\
Validated AUPR $p$ value (permutation test) & $<0.001$ \\
Precision threshold for network pruning & 0.32 \\
Edge score threshold for pruned network & 0.88242 \\
Number of edges (pruned/total) & $6836 / 211,410$ \\
Number of TFs (pruned/total) & $145 / 145$ \\
Number of targets (pruned/total) & $311 / 1458$ \\
\hline TF transcription factor, AUPR area under precision recall & \\
\hline
\end{tabular}

$\mathrm{TF}_{2}$ target edges for 116 TFs from the pruned DFG network (Supplementary Fig. 12 and Supplementary Data 10). Using this approach, we could link $76 \%$ of indirect TGA1 targets in planta (101/133) back to TGA1 through $49 / 63$ direct $\mathrm{TF}_{2}$ targets of TGA1. The set of 63 direct $\mathrm{TF}_{2}$ targets of TGA1 includes $13 \mathrm{TF}_{2} \mathrm{~s}$ whose direct regulated targets have been validated in TARGET (Fig. 2 and Supplementary Data 2), and $36 \mathrm{TF}_{2} \mathrm{~s}$ with highconfidence DFG predicted edges to indirect targets of TGA1. To further determine which of these intermediate $\mathrm{TF}_{2} \mathrm{~s}$ are most important in relaying the $\mathrm{N}$ signal downstream of TGA1, we used the 80 cis-motif clusters (Supplementary Fig. 10 and Supplementary Data 8 and 9) to perform analysis of CCM enrichment in TGA1 indirect targets. This analysis showed that the most enriched CCM in the TGA1 indirect targets corresponds to cluster 15 (NAC family) which is enriched in the gene body (FDR $=7.9 \mathrm{E}-5$, Fisher's exact test $)$ of TGA1 indirect targets.

We also performed a similar Network Walk for CRF4, and found that-by contrast to TGA1-relatively few $\mathrm{N} \times$ Time genes are directly controlled by CRF4 (65 genes), yet the 


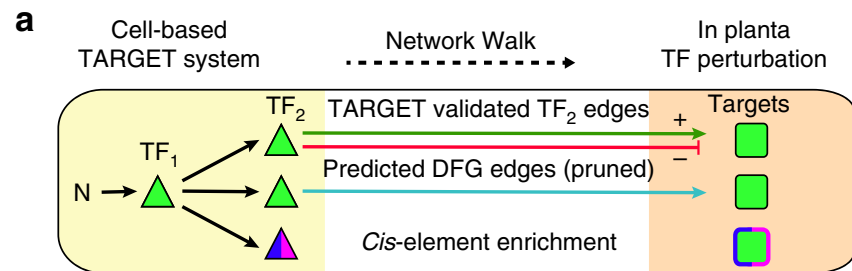

b
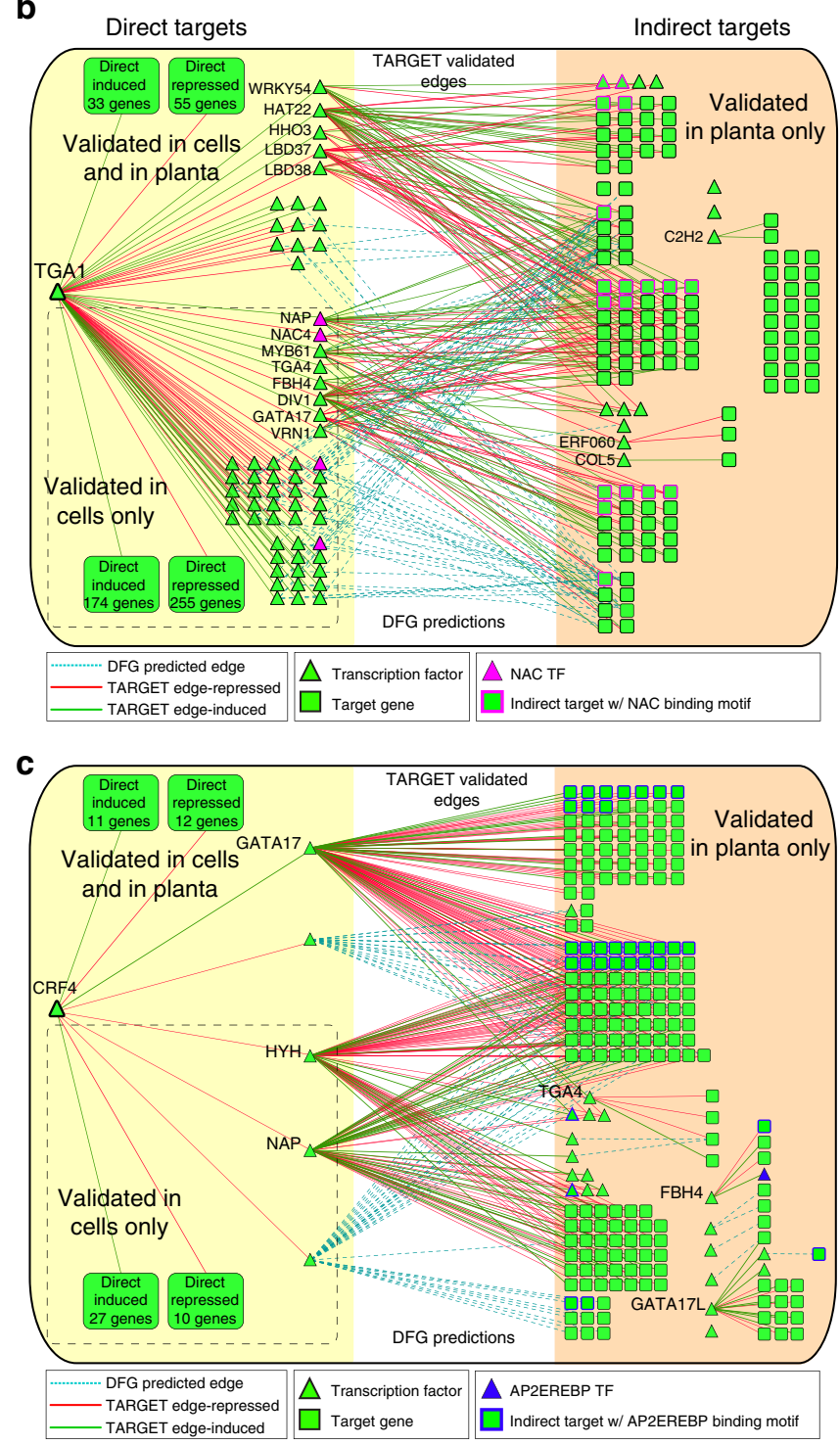

number of $\mathrm{N} \times$ Time root genes that respond to CRF4 overexpression in planta (247 genes) is similar to the number responding to TGA1 overexpression in planta (208 genes) (Fig. 7b, c). In the CRF4 Network Walk, we identified direct connections of CRF4 to only 5 intermediate $\mathrm{TF}_{2}$ s (GATA17, NAP, HYH, MYB34, bHLH112), which in turn accounted for the regulation of $87 \%$ of CRF4 indirect targets in planta. Thus, a Network Walk not only identifies the network path for each $\mathrm{TF}_{1}$ via its downstream $\mathrm{TF}_{2} \mathrm{~s}$, but can also help classify $\mathrm{TF}$ modes of action, as discussed below.

\section{Discussion}

An ultimate goal of systems biology is to learn GRNs and infer TF-target models that can accurately predict future gene
Fig. 7 Network Walking charts a path from direct to indirect transcription factor (TF) targets. a A schematic representation of the Network Walking approach used to connect direct TF targets identified in cells to the indirect targets identified only in planta. Example of Network Walks from direct targets identified in cells (yellow shaded region), to indirect targets identified only in planta (orange shaded region) for $\mathbf{b}$ TGA1 and c CRF4. Edges connecting the indirect targets back to TGA1/CRF4 through their direct $\mathrm{TF}_{2}$ targets come from validated TARGET (Transient Assay Reporting Genome-wide Effects of Transcription factors) edges as well as from high-confidence edges from the pruned time-inferred dynamic factor graph (DFG) network. Enrichment of the consensus cis-motif for the 80 clusters (Supplementary Fig. 10 and Supplementary Data 8) in the 500 bp promoters and gene body of the indirect targets of TGA1 and CRF4 was assessed. The most significant cluster onsensus cis-motif (CCM) in indirect targets of TGA1 was for cis-motif cluster 15 (NAC family) in the gene body. For CRF4, the CCM for cluster 8 (AP2EREBP) was enriched in the gene body of CRF4 indirect targets. The network shown is limited to TFs and targets that respond to $\mathrm{N} \times$ Time in Varala et al. ${ }^{29}$. For clarity, edges to target genes include only the top three validated edges based on fold change, and top ten predicted DFG edges based on edge score

expression states under untested conditions. A key step to accomplish this is the experimental validation of edges between TF regulators and their target genes to use in refinement or as priors in network inference. Our study addresses the challenge of identifying functional in vivo targets of a TF genome-wide in a rapid and medium-throughput manner. We also demonstrate that the direct regulated targets of a TF identified using TARGET can enhance our understanding of TF-DNA binding data. For example, we found that in vitro high-throughput methods for identifying TF binding can overestimate (DAP-seq ${ }^{5}$ ) or underestimate $\left(\mathrm{Y}_{1} \mathrm{H}^{28}\right)$ the number of functional $\mathrm{TF}$-target interactions identified using the TARGET assay (Supplementary Fig. 9 and Supplementary Data 3). Similarly, in vivo ChIP assays are a poor predictor of TF-mediated gene regulation ${ }^{8-10}$. We demonstrate how direct regulated TF-target edges can be used to filter network predictions. Specifically, we used 71,836 validated targets for $29 / 33 \mathrm{~N}$-early response TFs to prune a GRN predicted using $\mathrm{DFG}^{48}$ to obtain a refined $\mathrm{N} \times$ Time GRN, where $\sim 1 / 3$ rd of the edge predictions are likely true (Supplementary Fig. 12 and Supplementary Data 10). This approach enabled us extend our TARGET results beyond the 33 TFs to the remaining $116 \mathrm{~N} \times$ Time TFs for which we do not yet have validated edges, and identify which TFs to target for further study based on their relative influence on the $\mathrm{N} \times$ Time network.

To integrate our TARGET results with in planta data, we derived an approach called Network Walking (Fig. 7). The key feature of the Network Walking strategy is to connect the direct regulated $\mathrm{TF}_{1}$ edges identified in plant cells using TARGET to the indirect $\mathrm{TF}_{1}$ targets validated only in planta. This approach enabled us to identify the intermediate $\mathrm{TF}_{2} \mathrm{~S}$ important for mediating the signal between the initial $\mathrm{TF}_{1}$ and downstream in planta indirect targets. The results can guide combinatorial experiments (e.g., TF stacking) and validation experiments on important $\mathrm{TF}_{2} \mathrm{~s}$ that are identified in a systems biology cycle.

As proof of concept, we demonstrated the Network Walking approach for two TFs, TGA1 (Fig. 7b) and CRF4 (Fig. 7c), which are validated in planta regulators of the $\mathrm{N}$ response in Arabidopsis ${ }^{26-29}$. Our Network Walking approach showed that TGA1 directly regulates $40 \%(508 / 1458)$ of the $\mathrm{N} \times$ Time genes in roots, including $63 / 145 \mathrm{~N}$-responsive $\mathrm{TF}_{2} \mathrm{~s}$, amplifying the effect of TGA1 on the $\mathrm{N}$ response gene network. Moreover, our 
finding that CCM for the NAC family cluster is enriched in TGA1 indirect targets suggests that NAC TFs are particularly important $\mathrm{TF}_{2} \mathrm{~s}$ for propagating the $\mathrm{N}$ signal downstream of TGA1.

The second example of Network Walking was for CRF4 (Fig. 7c), a TF which was recently shown to be involved in $\mathrm{N}$ signaling in shoots and roots in planta ${ }^{29}$. The TARGET data showed that direct regulated CRF4 targets are specifically enriched for the shoot $\mathrm{N} \times$ Time-responsive genes (Fig. 2). Our Network Walk now resolves how CRF4 can have a significant influence on root N-responsive genes in planta (Supplementary Data 11), despite directly regulating only a small number of root $\mathrm{N} \times$ Time genes itself (Fig. 2). This is because CRF4 directly regulates $5 \mathrm{TF}_{2} \mathrm{~s}$ which collectively have edges validated by TARGET and/or high-confidence predicted DFG edges to $50 \%$ of the root $\mathrm{N} \times$ Time genes. Thus, the effect of CRF4 on roots in planta is mediated through these intermediate $\mathrm{TF}_{2} \mathrm{~s}$.

Overall, the 85,144 validated targets of $33 \mathrm{~N}$-early response TFs revealed a connected GRN regulating $88 \%$ of the genes that respond to $\mathrm{N}$ treatment as a function of time in whole roots and shoots $^{29}$, and the TFs collectively regulated a set of genes enriched in N-related processes (Fig. 3b, Supplementary Data 5). We also observed that the distribution of edges in the validated TF-target network (Fig. 3a) resembles a scale-free network ${ }^{51}$, a feature of biological networks that provides robustness ${ }^{52}$. Signal integration, an emergent property of biological systems ${ }^{53}$, could explain the large number of $\mathrm{N}$ responsive TFs in shoots and roots (258 TFs), roughly $10 \%$ of all the predicted Arabidopsis $\mathrm{TFs}^{29}$. Indeed, many of the $33 \mathrm{TFs}$ functionally validated in our study have defined roles in other pathways (Supplementary Data 12), linking the $\mathrm{N}$ response to other biological processes, including hormone signaling ${ }^{54}$ and biotic stress ${ }^{55}$. These types of complex combinatorial interactions between TFs, which integrate or fine-tune a response to signal inputs ${ }^{56}$, have been described in many organisms ${ }^{57-59}$.

Because TARGET is a cell-based TF perturbation system, direct regulated targets identified in vivo can provide biological context to TF-binding data. Somewhat surprisingly, our analysis revealed that all 33 of the TFs we assayed acted as both an inducer and repressor of direct regulated targets, and that cisbinding motifs for a TF are often specific to a particular direction of regulation (Fig. 4). Particularly interesting are group III TFs, where the pattern of enrichment of the known cis-motifs suggests that direct TF binding in the promoter leads to induction while direct binding to the gene body leads to repression. We also found that the direct regulated targets of TFs that could not be explained by TF binding to the known cis-motif for that TF (based on in vitro TF-DNA binding data) may involve partner TFs which are not present in in vitro binding assays. Indeed, our cis-motif cluster analysis (Fig. 5 and Supplementary Fig. 11) addressed the duality of TF function (e.g., inducer and repressor) observed in the regulatory action of each of the 33 TFs. We noted several instances where the direct TF targets were enriched in CCMs for TF families other than the TF under investigation (Fig. 5 and Supplementary Fig. 11). This finding could explain how the same TF could mediate repression of direct targets (via a partner TF-binding site) vs. induction when the TF binds directly to DNA. As one example, the cis-binding motif for NAP is enriched only in its induced targets (Fig. 4), while the repressed NAP targets are instead enriched in bHLH sites (clusters 6 and 36) in the $500 \mathrm{bp}$ promoter. Moreover, the experimentally validated interaction between NAP and a bHLH family TF-bHLH $96^{43}$ - could explain how NAP is able to repress its direct target genes via its interaction with bHLH96. This model is also supported by the finding that protein-protein interactions between TFs have been shown to alter the effect of a TF on target gene expression $^{60-62}$

The development of tools for validating the TF-target edges within inferred networks is crucial to obtaining high-quality predictive GRNs. In this study, we demonstrate that the TARGET system for cell-based TF perturbation complements existing TF-target binding approaches and in planta perturbation by providing direct TF-target edges based on TF-mediated gene regulation in cells isolated from the tissue of interest. Importantly, this system does not require the creation of stable transgenics and scales easily. We have also introduced an approach, Network Walking, which connects the early and direct regulated TF targets identified in cells using TARGET to downstream responses observed only in planta. While our proof-of-concept studies focused on rapid N signaling in Arabidopsis, both of these approaches are generally applicable to study of GRNs involved in transducing signals in any eukaryotic system in agriculture, biology, or medicine.

\section{Methods}

A scaled-up TARGET assay for TF perturbation in cells. To make the TFplasmid constructs, the $33 \mathrm{~N}$-early response TFs were TOPO cloned into pENTR (Invitrogen) from complementary DNA or isolated from the Arabidopsis TF collection $^{63}$. TFs were then transferred to the pBeaconRFP_GR plasmid ${ }^{18}$ or a GFP version of the same plasmid (pBeaconGFP_GR) by Gateway (Invitrogen) cloning.

For protoplasting and transfections, Arabidopsis Col-0 plants were grown in 1\% w/v sucrose, $0.5 \mathrm{~g}$ per L MES, $1 \times$ MS basal salts $(-\mathrm{CN}), 1 \mathrm{mM} \mathrm{KNO} \mathrm{KN}_{3}, 2 \%$ agar, $\mathrm{pH}$ 5.7 for 10 days prior to the TARGET experiment. Light conditions were $120 \mu \mathrm{mol}$ $\mathrm{m}^{-2} \mathrm{~s}^{-1}$ at constant temperature at $22^{\circ} \mathrm{C}, 16 \mathrm{~h}$ light, $8 \mathrm{~h}$ dark (long day). Roots of 10-day-old seedling were harvested and the cell wall removed using cellulase and macerozyme (Yakult, Japan) for $3 \mathrm{~h}$. Cells were filtered sequentially through $70 \mu \mathrm{m}$ and $40 \mu \mathrm{m}$ cell strainers (BD Falcon, USA) and pelleted at $500 \times g$. Filtered cells were washed with $15 \mathrm{~mL} \mathrm{MMg}$ solution (400 mM mannitol, $10 \mathrm{mM} \mathrm{MgCl}, 4 \mathrm{mM}$ MES pH 5.7), resuspended to approximately $2-3 \times 10^{6}$ cells per mL. For each transfection, in a $50 \mathrm{~mL}$ conical tube, $1 \mathrm{~mL}$ of cell suspension was mixed with 120 $\mu \mathrm{g}$ of plasmid DNA, $1 \mathrm{~mL}$ of PEG solution (40\% polyethylene glycol 4000 (Millipore Sigma, USA), $400 \mathrm{mM}$ mannitol, and $50 \mathrm{mM} \mathrm{CaCl}_{2}$ ) and vortexed gently for $5 \mathrm{~s}$. After mixing, $50 \mathrm{~mL}$ of W5 buffer $\left(154 \mathrm{mM} \mathrm{NaCl}, 125 \mathrm{mM} \mathrm{CaCl}_{2}, 5 \mathrm{mM}\right.$ $\mathrm{KCl}, 5 \mathrm{mM}$ MES, $5 \mathrm{mM}$ glucose, $\mathrm{pH}$ 5.7) was slowly added to the tube. Cells were pelleted at $1200 \times g$, and washed 3 times with W5 buffer. For each TF and the EV construct, 4-6 million cells were transfected and after washing, a single TF in the RFP vector and a single TF in the GFP vector were aliquoted into 3 replicate wells of a 24-well plate. After overnight incubation, each pool of transfected root protoplasts was treated with the $\mathrm{N}$ dose present in standard MS media ${ }^{64}(20 \mathrm{mM}$ $\mathrm{KNO}_{3}+20 \mathrm{mM} \mathrm{NH} \mathrm{NO}_{3}$ ) for $2 \mathrm{~h}$. Next, $35 \mu \mathrm{M} \mathrm{CHX}$ was added $20 \mathrm{~min}$ before a $10 \mu \mathrm{M}$ DEX treatment. Transfected cells were sorted by FACS into GFP- and RFPexpressing populations $3 \mathrm{~h}$ after DEX treatment.

For transcriptome analysis, cells expressing the candidate TF or EV were collected in triplicate and RNA-Seq libraries were prepared from their mRNA using the NEBNext ${ }^{\circledR}$ Ultra $^{\mathrm{TN}}$ RNA Library Prep Kit for Illumina ${ }^{\circledR}$. The RNA-Seq libraries were pooled (up to 27 libraries per run) and sequenced on the Illumina NextSeq 500 platform. The RNA-Seq reads were aligned to the TAIR10 genome assembly using TopHat $2^{65}$ and gene expression estimated using the GenomicFeatures/GenomicAlignments packages ${ }^{66}$. The gene counts for every sample were combined and DE genes in the TF transfected samples vs the EV samples were identified using the DESeq 2 package ${ }^{67}$ with a TF+Batch model and an FDR adjusted $p$ value $<0.05$. We filtered out genes that respond more than 5fold to CHX treatment in transfected protoplasts from the lists of TF targets (Supplementary Data 1 and Supplementary Methods). Genes that are expressed in any of the protoplast experiments (excluding the CHX-responsive genes in Supplementary Data 1) were used as the background for subsequent enrichment analyses.

Calculating nitrogen specificity and influence of TF targets. The specificity of each TF to target genes in the $\mathrm{N} \times$ Time-responsive dataset of Varala et al. ${ }^{29}$ was calculated by dividing the $\mathrm{N} \times$ Time-responsive targets of a TF by the total number of targets regulated by that TF. The influence of a TF on the $\mathrm{N} \times$ Time-responsive genes is the number of $\mathrm{N} \times$ Time-responsive genes targeted by a TF divided by the total number of $\mathrm{N} \times$ Time-responsive targets. The $\mathrm{N}$-specificity index $p$ value ${ }^{29}$ was calculated using the one proportion $z$-test to compare the proportion of targets for a $\mathrm{TF}$ in the genome to the proportion of targets for that $\mathrm{TF}$ in the root $\mathrm{N} \times \mathrm{Time}$ responsive genes ${ }^{29}$, under the null hypothesis that they are equal.

GO enrichment in TF-target networks. The web application agriGO v2.0 ${ }^{68}$ was used to identify GO terms enriched in the cumulative direct regulated targets of the 
33 TFs. To calculate the GO term enrichment in the sets of targets regulated by increasing number of TFs (Fig. 3b), let $G$ be a list of genes associated with a GO term and $T_{k}$ be a list of targets that are targeted by at least $k$ TFs. We can calculate the enrichment of $G\left(E_{g}\right)$ in $T_{k}$ by simply comparing the frequency of $G$ in $T_{k}$ (i.e., the fraction of genes in $T_{k}$ that intersect $G$ ), represented as $F_{k}$, to the frequency of $G$ in the background (all 20,662 genes expressed in protoplast experiments), represented as $F_{b}$.

$$
E_{g}=\frac{F_{k}}{F_{b}}
$$

To test for the significance of this enrichment, we used the figure of merit Focus. For each gene list $(G)$ we calculate Focus $\left(F_{g}\right)$ by simply adding the $E_{g}$ for all $k$ up to $n$ TFs:

$$
F_{g}=\sum_{k=1}^{n} \frac{F_{k}}{F_{b}}
$$

To test if the Focus of the validated network is significant for a given GO term, a permutation test was used to determine an empirical $p$ value by comparing the Focus of the validated network to the Focus of 1000 iterations of a randomized network, generated by shuffling the edges within the experimentally validated TF-target network.

Cis-motif enrichment and clustering. Enrichment of the cis-binding for motifs in TF target genes was calculated using the AME tool within the MEME package ${ }^{69}$. The background used corresponded to the same gene region for all genes expressed in any of the cell-based TARGET experiments and the frequency of bases to the base frequency within the background.

For cis-motif clustering, cis-binding motifs for Arabidopsis transcription factors were collected from DAP-seq ${ }^{5}$, Cis-BP ${ }^{38}$, PBM of Franco-Zorrilla et al. ${ }^{39}$ and ChIP-seq from Song et al. ${ }^{37}$. PWMs were converted to the MEME motif format ${ }^{70}$ and the RSAT matrix-clustering tool ${ }^{41}$ was used with the following parameters: hclust_method $=$ average, calc $=$ sum, metric_build_tree $=$ Ncor, lth w 5 lth cor $=$ 0.6 , lth Ncor $=0.45$, quick=true. To search for the enrichment of each cis-motif in the TF targets, the consensus PWM for each of the 80 cis-motif clusters was converted to the MEME format and the FIMO tool within the MEME package ${ }^{69}$ was used to identify every occurrence of each of the 80 consensus cis-motifs in the $500 \mathrm{bp}$ promoters and gene body of all 20,662 protoplast expressed genes at a $p$ value $<0.0001$. Overlapping cis-motifs were removed, retaining only the cis-motif with the lowest $p$ value. For each set of TF targets, enrichment of a cis-motif in the target set relative to their occurrence in all annotated genes was calculated using Fisher's exact test. The resulting $p$ values were FDR corrected. Heatmaps and hierarchical clustering were generated with Euclidean distance and the ward.D agglomeration method using the gplots heatmap. 2 function in R.

Identification of direct and indirect TGA1 targets in planta. The in planta TGA1 overexpression construct was made by Gibson assembly (NEB) with a three-part construct. The CaMV-35s promoter was fused to the TGA1 CDS using in the pGreen vector backbone. Primers used in the assembly are in Supplementary Table 4.

Arabidopsis Col-0 plants with the 35S:TGA1 transgene were generated using Agrobacterium-mediated floral-dip method. Approximately 100 seeds were sown in Phytatrays (Sigma-Aldrich) in liquid media that was identical to what was used in the TARGET assay: $1 \% \mathrm{w} / \mathrm{v}$ sucrose, $0.5 \mathrm{~g}$ per L MES, $1 \times$ MS basal salts $(-\mathrm{CN})$, $1 \mathrm{mM} \mathrm{KNO}_{3}$, pH 5.7. Light conditions were $120 \mu \mathrm{mol} \mathrm{m}^{-2} \mathrm{~s}^{-1}$ at constant temperature at $22^{\circ} \mathrm{C}, 16 \mathrm{~h}$ light, $8 \mathrm{~h}$ dark (long day).

When 35S:TGA1 seedlings were 13 days old, they were transferred to $\mathrm{N}$ starvation media $(1 \% \mathrm{w} / \mathrm{v}$ sucrose, $0.5 \mathrm{~g}$ per L MES, $1 \times$ MS basal salts $(-\mathrm{CN}), \mathrm{pH}$ 5.7). After $24 \mathrm{~h}$, at $2 \mathrm{~h}$ after subjective dawn, seedlings were transferred to Phytatrays containing identical media with the addition of the $\mathrm{N}$ dose in standard MS media ${ }^{64} 20 \mathrm{mM} \mathrm{KNO}_{3}+20 \mathrm{mM} \mathrm{NH}_{4} \mathrm{NO}_{3}$ or $20 \mathrm{mM} \mathrm{KCl}$ control. Plants were incubated within treatment media for $2 \mathrm{~h}$ after which root tissue was immediately harvested and flash frozen in liquid nitrogen.

RNA was extracted from root tissue using the QIAGEN RNeasy kit (Qiagen). mRNA was purified with oligo-dT beads (Invitrogen), and RNA-seq libraries made using the NEBNext Ultra Library Prep Kit (NEB). Libraries were sequenced the Illumina HiSeq 2500 v4 platform using $1 \times 50$ or $1 \times 75$ single end chemistry. RNAseq reads were aligned as described for the protoplast samples and DE genes were identified using DESeq2 ${ }^{67}$

Time-based network inference and AUPR validation. The time-based DFG network inference 48 predicted GRN was generated using the N-treatment timeseries data as described in Supplementary Methods. We used a pruning approach to filter this network for high-confidence edges ${ }^{1}$. The validated TF-target edges from TARGET were used to perform an AUPR analysis and identify a precision threshold of 0.32 (Fig. 6 and Table 2). This TF-target edge cut-off was chosen to minimize false positives, while recovering as many true positives as possible. The resulting pruned DFG inferred network was visualized (Supplementary Fig. 12) using Cytoscape ${ }^{71}$. Precision, recall, and $F$-score were calculated for the edges in the pruned network to generate Supplementary Data 10.

$$
\begin{gathered}
\text { Precision }=\text { True Positives } /(\text { True Positives }+ \text { False Positives }) . \\
\text { Recall }=\text { True Positives } /(\text { True Positives }+ \text { False Negatives }) . \\
\mathrm{F}-\text { score }=(2 * \text { Precision } * \text { Recall }) /(\text { Precision }+ \text { Recall }) .
\end{gathered}
$$

Reporting summary. Further information on experimental design is available in the Nature Research Reporting Summary linked to this article.

\section{Data availability}

All raw sequencing data from this project have been deposited in the Gene Expression Omnibus (GEO) database accession GSE117857 and GSE128209. Data supporting the findings of this work are available within the paper and its Supplementary Information files. A reporting summary for this Article is available as a Supplementary Information file. The datasets generated and analyzed during the current study are available from the corresponding author on reasonable request. The source data underlying Figs. 3a, 3b, 5a 5b, and 6, as well as Supplementary Figs. 2, 4b-d, 5, 6, 8, 9, 11a, and $11 \mathrm{~b}$ are provided as a Source Data file.

\section{Code availability}

The previously published dynamic factor graphs (DFG) network inference machinelearning code is available at [https://github.com/piotrmirowski/DFG4GRN] ${ }^{49}$. Python scripts used for calculation of motif enrichment and randomization the networks for calculating $p$ values are available from the corresponding author upon reasonable request.

Received: 29 July 2018 Accepted: 15 March 2019

Published online: 05 April 2019

\section{References}

1. Marbach, D. et al. Wisdom of crowds for robust gene network inference. Nat Methods 9, 796 (2012).

2. Li, Y., Pearl, S. A. \& Jackson, S. A. Gene networks in plant biology: approaches in reconstruction and analysis. Trends Plant. Sci. 20, 664-675 (2015).

3. Basso, K. et al. Reverse engineering of regulatory networks in human B cells. Nat. Genet. 37, 382 (2005).

4. Arrieta-Ortiz, M. L. et al. An experimentally supported model of the Bacillus subtilis global transcriptional regulatory network. Mol. Syst. Biol. 11, 839 (2015).

5. O'Malley, R. C. et al. Cistrome and epicistrome features shape the regulatory DNA landscape. Cell 166, 1598 (2016).

6. Bulyk, M. L. Protein binding microarrays for the characterization of DNAprotein interactions. Adv. Biochem. Eng. Biotechnol. 104, 65-85 (2007).

7. Breton, G., Kay, S. A. \& Pruneda-Paz, J. L. in Environmental Responses in Plants: Methods and Protocols (ed. Paula Duque) 107-118 (Springer, New York, 2016).

8. Gitter, A. et al. Backup in gene regulatory networks explains differences between binding and knockout results. Mol. Syst. Biol. 5, 276 (2009).

9. Marchive, C. et al. Nuclear retention of the transcription factor NLP7 orchestrates the early response to nitrate in plants. Nat. Commun. 4, 1713 (2013).

10. Swift, J. \& Coruzzi, G. M. A matter of time - How transient transcription factor interactions create dynamic gene regulatory networks. Biochim. Biophys. Acta 1860, 75-83 (2017).

11. Kuo, M.-H. \& Allis, C. D. In vivo cross-linking and immunoprecipitation for studying dynamic protein: DNA associations in a chromatin environment. Methods 19, 425-433 (1999).

12. Cuvier, O. \& Fierz, B. Dynamic chromatin technologies: from individual molecules to epigenomic regulation in cells. Nat. Rev. Genet. 18, 457 (2017).

13. Todeschini, A.-L., Georges, A. \& Veitia, R. A. Transcription factors: specific DNA binding and specific gene regulation. Trends Genet. 30, 211-219 (2014)

14. Li, Y., Varala, K. \& Coruzzi, G. M. From milliseconds to lifetimes: tracking the dynamic behavior of transcription factors in gene networks. Trends Genet. 31, 509-515 (2015)

15. Cantone, I. et al. A yeast synthetic network for in vivo assessment of reverse engineering and modeling approaches. Cell 137, 172-181 (2009).

16. Vermeirssen, V., De Clercq, I., Van Parys, T., Van Breusegem, F. \& Van de Peer, Y. Arabidopsis ensemble reverse-engineered gene regulatory network discloses interconnected transcription factors in oxidative stress. Plant Cell 26, 4656-4679 (2014) 
17. Faith, J. J. et al. Large-scale mapping and validation of Escherichia coli transcriptional regulation from a compendium of expression profiles. PLoS Biol. 5, e8 (2007).

18. Bargmann, B. O. et al. TARGET: a transient transformation system for genome-wide transcription factor target discovery. Mol Plant 6, 978-980 (2013).

19. Para, A. et al. Hit-and-run transcriptional control by bZIP1 mediates rapid nutrient signaling in Arabidopsis. Proc. Natl. Acad. Sci. USA 111, 10371-10376 (2014)

20. Medici, A. et al. AtNIGT1/HRS1 integrates nitrate and phosphate signals at the Arabidopsis root tip. Nat. Commun. 6, 6274 (2015).

21. Doidy, J. et al. "Hit-and-Run" transcription: de novo transcription initiated by a transient bZIP1 "hit" persists after the "run". BMC Genom. 17, 92 (2016).

22. Sparks, E. E. et al. Establishment of expression in the SHORTROOTSCARECROW transcriptional cascade through opposing activities of both activators and repressors. Dev. Cell 39, 585-596 (2016)

23. Codamo, J., Munro, T. P., Hughes, B. S., Song, M. \& Gray, P. P. Enhanced $\mathrm{CHO}$ cell-based transient gene expression with the epi-CHO expression system. Mol. Biotechnol. 48, 109-115 (2011).

24. Huynh, C. Q. \& Zieler, H. Construction of modular and versatile plasmid vectors for the high-level expression of single or multiple genes in insects and insect cell lines. J. Mol. Biol. 288, 13-20 (1999).

25. Yin, J., Li, G., Ren, X. \& Herrler, G. Select what you need: a comparative evaluation of the advantages and limitations of frequently used expression systems for foreign genes. J. Biotechnol. 127, 335-347 (2007).

26. Alvarez, J. M. et al. Systems approach identifies TGA1 and TGA4 transcription factors as important regulatory components of the nitrate response of Arabidopsis thaliana roots. Plant J. 80, 1-13 (2014).

27. Canales, J., Contreras-López, O., Álvarez, J. M. \& Gutiérrez, R. A. Nitrate induction of root hair density is mediated by TGA1/TGA4 and CPC transcription factors in Arabidopsis thaliana. Plant J. 92, 305-316 (2017).

28. Gaudinier, A. et al. Transcriptional regulation of nitrogen-associated metabolism and growth. Nature 563, 259-264 (2018).

29. Varala, K. et al. Temporal transcriptional logic of dynamic regulatory networks underlying nitrogen signaling and use in plants. Proc. Natl. Acad. Sci. USA 115, 6494-6499 (2018).

30. Vidal, E. A., Alvarez, J. M. \& Gutierrez, R. A. Nitrate regulation of AFB3 and NAC4 gene expression in Arabidopsis roots depends on NRT1.1 nitrate transport function. Plant Signal. Behav. 9, e28501 (2014).

31. Rubin, G., Tohge, T., Matsuda, F., Saito, K. \& Scheible, W. R. Members of the LBD family of transcription factors repress anthocyanin synthesis and affect additional nitrogen responses in Arabidopsis. Plant Cell 21, 3567-3584 (2009).

32. Maeda, Y. et al. A NIGT1-centred transcriptional cascade regulates nitrate signalling and incorporates phosphorus starvation signals in Arabidopsis. Nat. Commun. 9, 1376 (2018).

33. Castaings, L. et al. The nodule inception like protein 7 modulates nitrate sensing and metabolism in Arabidopsis. Plant J. 57, 426-435 (2009).

34. Aoyama, T. \& Chua, N. H. A glucocorticoid-mediated transcriptional induction system in transgenic plants. Plant J. 11, 605-612 (1997).

35. Yamaguchi, N., Winter, C. M., Wellmer, F. \& Wagner, D. Identification of direct targets of plant transcription factors using the GR fusion technique. Methods Mol. Biol 1284, 123-138 (2015).

36. Birkenbihl, R. P., Kracher, B., Roccaro, M. \& Somssich, I. E. Induced genomewide binding of three Arabidopsis WRKY transcription factors during early MAMP-triggered immunity. Plant Cell 29, 20-38 (2017).

37. Song, L. et al. A transcription factor hierarchy defines an environmental stress response network. Science 354, aag1550 (2016).

38. Weirauch, M. T. et al. Determination and inference of eukaryotic transcription factor sequence specificity. Cell 158, 1431-1443 (2014).

39. Franco-Zorrilla, J. M. et al. DNA-binding specificities of plant transcription factors and their potential to define target genes. Proc. Natl. Acad. Sci. USA 111, 2367-2372 (2014).

40. Sullivan, AlessandraM. et al. Mapping and dynamics of regulatory DNA and transcription factor networks in A. thaliana. Cell Rep. 8, 2015-2030 (2014).

41. Castro-Mondragon, J. A., Jaeger, S., Thieffry, D., Thomas-Chollier, M. \& van Helden, J. RSAT matrix-clustering: dynamic exploration and redundancy reduction of transcription factor binding motif collections. Nucleic Acids Res. 45, e119 (2017).

42. Khan, A. et al. JASPAR 2018: update of the open-access database of transcription factor binding profiles and its web framework. Nucleic Acids Res. 46, D260-D266 (2018).

43. Trigg, S. A. et al. CrY2H-seq: a massively multiplexed assay for deep-coverage interactome mapping. Nat. Methods 14, 819-825 (2017).

44. Song, Y. H. et al. Isolation of CONSTANS as a TGA4/OBF4 interacting protein. Mol. Cells 25, 559-565 (2008).

45. Datta, S. et al. LZF1/SALT TOLERANCE HOMOLOG3, an Arabidopsis Bbox protein involved in light-dependent development and gene expression, undergoes COP1-mediated ubiquitination. Plant Cell 20, 2324-2338 (2008).
46. Arabidopsis Interactome Mapping Consortium. Evidence for network evolution in an Arabidopsis interactome map. Science 333, 601-607 (2011).

47. Husbands, A., Bell, E. M., Shuai, B., Smith, H. M. S. \& Springer, P. S. LATERAL ORGAN BOUNDARIES defines a new family of DNA-binding transcription factors and can interact with specific bHLH proteins. Nucleic Acids Res. 35, 6663-6671 (2007).

48. Mirowski, P. \& LeCun, Y. Dynamic factor graphs for time series modeling. Lect. Notes Artif. Int. 5782, 128-143 (2009).

49. Krouk, G., Mirowski, P., LeCun, Y., Shasha, D. E. \& Coruzzi, G. M. Predictive network modeling of the high-resolution dynamic plant transcriptome in response to nitrate. Genome Biol. 11, R123 (2010)

50. Hase, T., Ghosh, S., Yamanaka, R. \& Kitano, H. Harnessing diversity towards the reconstructing of large scale gene regulatory networks. PLoS Comput. Biol. 9, e1003361 (2013).

51. Barabási, A.-L. \& Albert, R. Emergence of scaling in random networks. Science 286, 509-512 (1999).

52. Albert, R. Scale-free networks in cell biology. J. Cell Sci. 118, 4947-4957 (2005).

53. Bhalla, U. S. \& Iyengar, R. Emergent properties of networks of biological signaling pathways. Science 283, 381-387 (1999)

54. Ristova, D. et al. Combinatorial interaction network of transcriptomic and phenotypic responses to nitrogen and hormones in the Arabidopsis thaliana root. Sci. Signal. 9, rs13-rs13 (2016).

55. Farjad, M. et al. Nitrogen limitation alters the response of specific genes to biotic stress. Int. J. Mol. Sci. 19, 3364 (2018).

56. Buchler, N. E., Gerland, U. \& Hwa, T. On schemes of combinatorial transcription logic. Proc. Natl. Acad. Sci. USA 100, 5136-5141 (2003).

57. Knight, H. \& Knight, M. R. Abiotic stress signalling pathways: specificity and cross-talk. Trends Plant. Sci. 6, 262-267 (2001).

58. Dolmetsch, R. E., Lewis, R. S., Goodnow, C. C. \& Healy, J. I. Differential activation of transcription factors induced by $\mathrm{Ca} 2+$ response amplitude and duration. Nature 386, 855 (1997).

59. Chen, $X$. et al. Integration of external signaling pathways with the core transcriptional network in embryonic stem cells. Cell 133, 1106-1117 (2008).

60. Harbison, C. T. et al. Transcriptional regulatory code of a eukaryotic genome Nature 431, 99 (2004)

61. Gerstein, M. B. et al. Architecture of the human regulatory network derived from ENCODE data. Nature 489, 91 (2012).

62. Boer, D. R. et al. Structural basis for DNA binding specificity by the auxindependent ARF transcription factors. Cell 156, 577-589 (2014).

63. Pruneda-Paz, J. L. et al. A genome-scale resource for the functional characterization of Arabidopsis transcription factors. Cell Rep. 8, 622-632 (2014).

64. Murashige, T. \& Skoog, F. A revised medium for rapid growth and bio assays with tobacco tissue cultures. Physiol. Plant. 15, 473-497 (1962).

65. Kim, D. et al. TopHat2: accurate alignment of transcriptomes in the presence of insertions, deletions and gene fusions. Genome Biol. 14, R36 (2013).

66. Lawrence, M. et al. Software for computing and annotating genomic ranges. PLoS Comput. Biol. 9, e1003118 (2013).

67. Love, M. I., Huber, W. \& Anders, S. Moderated estimation of fold change and dispersion for RNA-seq data with DESeq2. Genome Biol. 15, 550 (2014)

68. Tian, T. et al. agriGO v2.0: a GO analysis toolkit for the agricultural community, 2017 update. Nucleic Acids Res. 45, W122-W129 (2017).

69. McLeay, R. C. \& Bailey, T. L. Motif Enrichment Analysis: a unified framework and an evaluation on ChIP data. BMC Bioinforma. 11, 165 (2010).

70. Bailey, T. L. et al. MEME SUITE: tools for motif discovery and searching. Nucleic Acids Res. 37, W202-208 (2009).

71. Shannon, P. et al. Cytoscape: a software environment for integrated models of biomolecular interaction networks. Genome Res. 13, 2498-2504 (2003).

\section{Acknowledgements}

We thank Dr. Daniel Tranchina, Dr. Carol Huang, and Dr. Manpreet Katari for their input and discussions regarding various analyses, and Dr. Laurie Leonelli for her very critical reading of the manuscript. This work was supported by NIH Grant GM032877 to G.M.C., NIH NIGMS Fellowship F32GM116347 to M.D.B., a Plant Genomics Grant from the Zegar Family Foundation (A160051) to G.M.C., and in part by the Laboratoire International Associé (LIA-CoopNet) funded by the Centre National de Recherche Scientifique (CNRS) to G.K. and G.M.C.

\section{Author contributions}

M.D.B, J.S., K.V., R.A.G., G.K, D.S., and G.M.C. designed research; M.D.B, J.C., A.V.P, J S., J.M.A., and S.M. performed research; C.-L.J. contributed new analytical tools; M.D.B. J.C., and J.S. analyzed the data; and M.D.B., D.S. and G.M.C. wrote the paper. 


\section{Additional information}

Supplementary Information accompanies this paper at https://doi.org/10.1038/s41467019-09522-1.

Competing interests: The authors declare no competing interests.

Reprints and permission information is available online at http://npg.nature.com/ reprintsandpermissions/

Journal Peer Review Information: Nature Communications thanks Rainer Spang and other anonymous reviewer(s) for their contribution to the peer review of this work.

Publisher's note: Springer Nature remains neutral with regard to jurisdictional claims in published maps and institutional affiliations. (c) (i) Open Access This article is licensed under a Creative Commons Attribution 4.0 International License, which permits use, sharing, adaptation, distribution and reproduction in any medium or format, as long as you give appropriate credit to the original author(s) and the source, provide a link to the Creative Commons license, and indicate if changes were made. The images or other third party material in this article are included in the article's Creative Commons license, unless indicated otherwise in a credit line to the material. If material is not included in the article's Creative Commons license and your intended use is not permitted by statutory regulation or exceeds the permitted use, you will need to obtain permission directly from the copyright holder. To view a copy of this license, visit http://creativecommons.org/ licenses/by/4.0/.

(C) The Author(s) 2019 